\title{
New Fuzzy Bus Signal Priority Control System Design Based on Wireless Sensor Networks
}

\author{
Yang Wang*
}

School of Electronic and Communication Engineering, Shenzhen Polytechnic, Shenzhen, 518055, China

\begin{abstract}
Bus rapid transit (BRT) is one of most effective methods to optimize urban traffic. Transit signal priority (TSP) is very important to increase the efficiency of BRT. TSP provides priority signals for public transport vehicles based on the advanced inspection and communication system. In this paper, TSP control system is designed with wireless sensor networks, which can support real-time information exchanging and bus positioning function through critical time synchronization. Taking into consideration that TSP maybe interfere other vehicles when giving the priority to public transport vehicles, this paper puts forward a fuzzy control algorithm which decides what kinds of traffic signals support based on the bus information. And this algorithm can reduce average vehicle delay at intersection, and realize the optimal control balance between bus priority and other vehicles.
\end{abstract}

Keywords: Bus rapid transit, fuzzy control, time synchronization, transit signal priority, wireless sensor networks.

\section{INTRODUCTION}

BRT (Bus Rapid Transit) system is one new method after the traditional mode of public transport mode and rail transport. BRT takes the public transport private road space, which can keep not only the characteristics of rail transit but also the flexibility, the convenience and the high speed of public transportation. The required of BRT system construction investment is about 10 times lower than the rail transit needed, whose construction period is shorter and implementation is more flexible. In fact, the number of passengers bus taken is almost the same as the light rail transit system, but the cost of bus is more cheaper. BRT effectively inhibits the number of passengers decline because of the increasing of private cars, which can guide the urban development $[1,2]$.

BRT lanes setting, and how to give signal priority to BRT vehicles at the intersection and suitable stops, and intelligent charging system are the important areas of BRT study. But the transit signal priority (TSP) now is becoming more important research issue than the others. In city, Urban Road will inevitably encounter a variety of signal-controlled intersection $[3,4]$. Data shows that intersection delay time of the tour accounted for $10-20 \%$ of whole buses travel time, which accounted for $50 \%$ of the whole delay. Therefore the bus signal priority control of BRT in intersection is most important for realization of fast, punctual and reliable BRT. TSP has shown promise in improving the performance of public transport vehicles by ensuring schedule adherence and reducing delay and operational cost. The simulation approach has commonly been used for evaluating TSP strategies as well as their impacts on other vehicles $[5,6]$. Basic requirements for researching TSP involve studying the logic of traffic signals both under normal operation and during transit signal priority, detection of a bus at the check-in and check-out points, priority generator controller, priority server controller, wireless communication between buses and traffic signals controllers, bus movements in the traffic stream, and dwell time at bus stops $[7,8]$.

In the latest intelligent transportation systems conference reports [9-11], it is concluded that Early Green and Extended Green are the two most commonly applied operational strategies in such transit signal priority systems. These two strategies both rely on shortening the green light time of the opposing approaches to obtain extra green light time for the prioritized approach $[12,13]$.

But with the development wireless sensor networks, especially ZigBee and RFID, real-time transit signal priority system research is proposed [14-16]. This paper presents wireless sensor networks system which can realize information exchanging between buses and traffic signal controllers, and give the buses position to priority generators. In addition, this paper puts forward one fuzzy control algorithm which can decide which kinds of traffic signals giving to ensure the optimal control balance between buses priority and other vehicles.

\section{BRT CONTROL ANALYSIS}

\subsection{Passive Priority Control Strategy}

Passive priority control strategy can reduce bus delays at intersections through the fixed signal timing, the passive priority control strategies are as follows:

(1) Adjusting the period of traffic signal to increase the effective green light for the lane of public transport vehicles. 
This method can reduce probability when bus meets red light at intersections, and then reduce the waiting time of public transport vehicles.

(2) Shortening signal period and compressing phase time to reduce the period time of buses waiting for the green light. But this method will reduce the capacity of the whole intersections.

(3) The green light of buses lane adopts phase separation strategy. This approach is similar to shorten the signal cycle. The principle is keeping the cycle and phase time constant, and then separating the phase of the green light of buses lanes and inserting the phase of the green light into the adjacent lanes' phases.

\subsection{Active Priority Control Strategy}

Active priority signals overcome the defects of signal loss in the passive control strategy through adjusting the traffic signal real-timely and dynamically. There are three main strategies including extending green light period, reducing red light period, and signal phase inserting function.

\section{(1) Strategy of extending the green light period}

When the bus arrives at intersection by the end of the green light period, the green light period of the bus's phase will be extended to ensure the bus can pass the intersection successfully. But the green light can't be extended indefinitely, because there is the maximum limit for the extended green light. When the green's maximum limit reached, the current phase of the green light should be forced to end, and then the phase will be converted. The maximum bus green light of phase is according to the green which is from the phase signal control calculation.

\section{(2) Strategy of shortening the red light period}

If the bus who satisfies the priority condition reaches the intersection when the red light, the current phase could be shortened, so that the arrival of the next green light phase will be advanced and bus priority will be realized. But the red light should not be allowed to shorten at any time, because there is a minimum of red light time. Minimum of red light time should be determined based on consideration about the minimum time required for pedestrians crossing the intersection.

(3) Strategy of inserting into the bus phase

If when the bus reached during the red period, furthermore there is a long time waiting for the green light start, then a special bus phase could be inserted for the bus, and after that the phase will be restored to the original. Signal phase insertion will lead to more loss of signal time, and then intersection capacity reduces much.

For multi-phase signal intersection, if the public transport vehicles from two directions have the same priority, and both directions are all red light signal phase, then the signal phase in accordance with pre-set sequence of operation. If one of the buses' next phase is the green light, this direction will compress the period of the red. The impact from the insertion phase of bus priority strategies should be reduced as far as possible.

\section{(4) Strategy of Real-time priorities control}

Real-time priority control is developed recently. It can optimize transport efficiency, the impact of other transport can be reduced to the lowest, when the bus delay can be reduced and the travel time can be shortened at the same time.

\section{INTELLIGENT CONTROL STRATEGY OF SIG- NAL CONTROLLER DESIGN}

This paper designed one real-time bus signal priority control system based on wireless sensor networks. In this paper, wireless sensor networks is not only used to exchange the message between bus and real-time bus signal priority control system, but also to give the location information of bus to the control system.

Signal controller constantly receives the data packets from monitoring center. Preprocessing module process information in multiple data packets at first then get input of intelligent decision-making module (phase priority); intelligent decision-making module decides the signal control status to ensure that bus has the priority by comparing the priorities of different phases, considering the other traffic flow information, then to output the result in the form of signal lights through the module.

Pre-processing can be divided into two stages:

(1) In the first stage, data processing for vehicles is completed to get the phase information of vehicles and vehicle passage priority.

(2) In the second stage, to combine the data in the same phase, then to get the passage priority of each phase.

In the first stage, the pre-processing module compare the receiving time of the information at first, then take the latest data packet for processing. The phase information of the vehicle can be found, which can be obtained by inquiring the identification information of fixed unit, the number of buses and buses' departure time.

Priority to the passage of vehicles can be obtained by calculating the extent of delay and the passenger capacity. The extent of delay is the main factors and the passenger capacity is the minor. The bus priority can be calculated through the specialized algorithm. The passenger capacity can be read directly from data packets.

The extent of delay is calculated by comparing the actual bus journey times and a standard bus journey times predetermined by experience to get a distinctions of time, so that the bus is early or late can be known when it arrives at that point. If it is late, the time of late should be calculated. To simplify the input data, the time of late should be quantized to get the extent of delay correspondingly. If not late, then ignoring this information, the purpose is to give priority 


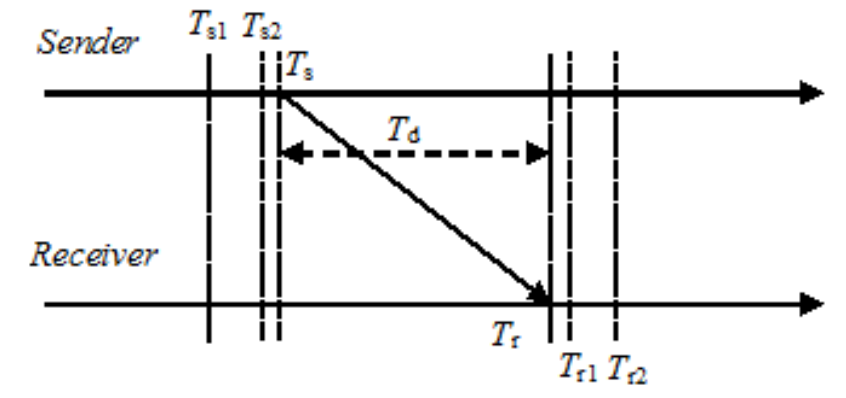

Fig. (1). Sender and receiver message time stamp.

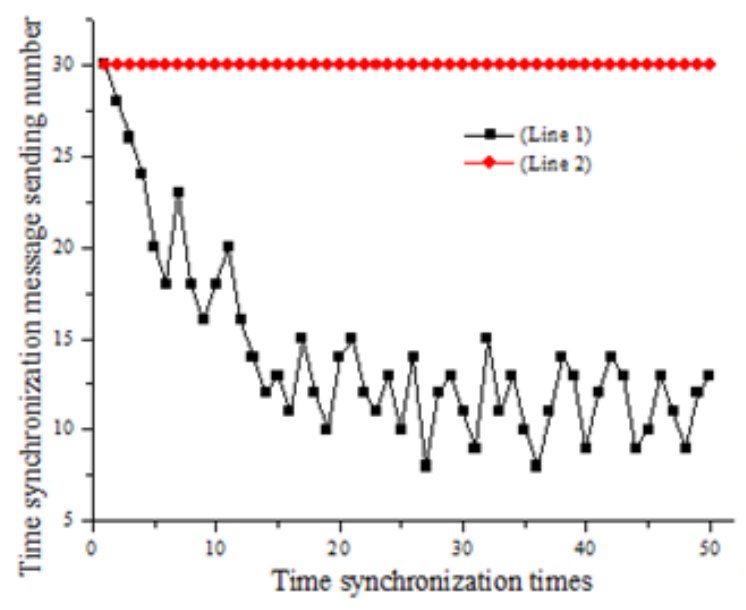

Fig. (2). 30 nodes FTSP application result.

to late buses only, so as to minimize the delay of other vehicles. In the second stage, the pre-processing module classify all vehicles according to the phase information at first, then calculate the pass priority of each phase according to priority of different vehicles. The calculation methods can be simple accumulation, and also can be accumulated after weighted. For example, when a bus has higher passage priority than the other buses at the cross, the weight of the vehicle should be increased when calculating.

\subsection{Time Synchronization}

To ensure the wireless sensor networks communicate in time, this paper adopts one improved Flooding Time Synchronization Protocol (FTSP). FTSP belongs to senderreceiver protocol, so sender which is based on the technology of MAC layer timestamp embedded in the sending moment, while receiver also records the receiving moment in the MAC layer. Differing from other sender-receiver synchronization protocol, in the FTSP protocol, sender marks several timestamps sequentially when sending a message. According to these timestamps, receiver could estimate the time delayed by the interrupt. The receiver could compensate for the receiving time according to the time of the interrupt delay and coding and decoding time, and then more precise synchronization points will be obtained (the time stamp in Fig. 1), as follows:
- After completion of sending preamble bytes, the FTSP algorithm marks the timestamp (Ts) in time synchronization message and transmits. The timestamp Ts is the current time minus the transmitting time of data part which including timestamp Ts, which can be obtained by the length of data and the speed of transmitting. Ts1 and Ts2 mean the multiple time stamps in sender.

- The receiver records the last bit of preamble bytes reaching time (Tr), and calculates the bit offset. After the complete message received, the receiver calculates the time delay $\mathrm{Td}$ through bit offset, which can be calculated by bit offset and the speed of receiving. $\operatorname{Tr} 1$ and $\operatorname{Tr} 2$ mean the multiple time stamps in receiver.

- The receiver calculates the time deviation from the sender.

In Fig. (2), we placed 30 wireless sensor nodes uniformly distributed in 5000 square meters to simulate the real intersection situation.

In the experiment, we can see that, the improved FTSP can reduce the number of time synchronization message sending greatly than the original FTSP, which can reduce the probabilities of network storm efficiently. This will be very helpful to ensure the real-time message exchanging. 
Table 1. RSSI value of distance.

\begin{tabular}{|c|c|}
\hline Distance/m & RSSI/dBm \\
\hline \hline 1 & -16 \\
\hline 3 & -25 \\
\hline 5 & -31 \\
\hline 10 & -41 \\
\hline 15 & -61 \\
\hline 20 & -63 \\
\hline 30 & -75 \\
\hline
\end{tabular}

\subsection{Location Information}

In the bus, the signal controller module will send information to monitoring center through wireless sensor networks module. The monitoring center will receive the message and then the compute the received signal strength indication (RSSI) value, which can reflect the distance from bus to monitoring center. As the Table 1 shows, there is the relationship between the RSSI value and the distance.

\subsection{Fuzzy Logic Control Strategy}

Bus priority modules under the light of the current green light phase of the situation and public transport vehicles to be released under the red light phase of a public transportation vehicles waiting in line under the circumstances, to determine which phase of the priority public transport vehicles under the release. It is called the output of a priority P (Priority) of the variables, $\mathrm{P}$ said that under the current green light phase relative to public transport vehicles to be released under the red light phase of a public transport vehicles priority under the prevailing demand.

Bus priority module is a two-input single-output fuzzy decision-making mode blocks, inputs include: CWT and NWT, CWT said that the current green light. All sections of the phase-controlled intersection approach the nearest road in the distance. Bus in the intersection stop line to reach the time required for the value of their language as: $\mathrm{Z}$ (zero), $\mathrm{S}$ (short), M (in), L (long).

At the same time a number of buses in the road, then take the minimum time required. NWT for the next phase to be controlled by the release of the red light, all sections of passage into the road in the bus queue always tired now total waiting time, the language is: $\mathrm{Z}$ (zero), VS (very short), $\mathrm{S}$ (Short), M (in), L (long), VL (very long). If the phase simultaneously, buses have to wait in line, then take all the accumulated waiting bus. Output is $\mathrm{P}$, said the priority, the language is: NB (Negative big), NM (negative of), NS (negative small), Z (zero), PS (positive small), PM (middle), PB (CP). If the priority value is positive, then the table green light said the current phase of the section under the control of the bus moving into the road. Car to give priority to traffic, the greater the value of the bus reached the junction is the time. Shorter, that is, the greater priority; if the priority value is negative, then that the next phase to be released under the control of the red route in the road. Waiting in traffic to give priority to buses, the smaller the value that the phase have will wait longer, the greater priority.

Bus priority module input and output variables of the fuzzy partition which not described in detail, it is this fuzzy rule: $\mathrm{P}$ inverse in the CWT and NWT, that is, if the current green phase of the control sections of the road into the bus soon reached the junction, and (or) the red light on the next phase to be released under the control of the public road approach orthogonal waiting in line for a short time, the priority for the CP, should be appropriate when the green light to extend the time for the current phase of the road in the bus stop car through the intersection. Bus priority module has 17 fuzzy rules, and the Table 2 lists some of the rules.

\section{SUMMARY}

This paper presents a bus priority signal control system based on wireless sensor networks, on the basis of the fuzzy control decision making. The technology is not uniform signal period, from that point of view, can also reduce the average vehicle delay. The simulation results show the effectiveness of this method. When the private car usage can't be reduced effectively, we should focus on the bus priority network technology and coordination technology to combine public transport vehicles not only reduces the average travel time, and as far as possible not increase the delay of other social vehicles.

This control strategy is charged through the signal control unit within the region and coordination of information exchange, to achieve smooth flow of traffic within the region. In saturated traffic condition, the following may result in traffic congestion outside the region charged. Therefore, this control strategy and coordination of road traffic can be used in conjunction with the induction of better control effect. 
Table 2. Some rules of two bus priority modules.

\begin{tabular}{|c|c|c|c|}
\hline Rule & CWT & NWT & P \\
\hline \hline 1 & $\mathrm{Z}$ & $\mathrm{Z}$ & $\mathrm{PB}$ \\
\hline 2 & $\mathrm{~S}$ & $\mathrm{~V}$ & $\mathrm{~S}$ \\
\hline 3 & $\mathrm{~S}$ & $\mathrm{M}$ & $\mathrm{PM}$ \\
\hline 4 & $\mathrm{~S}$ & $\mathrm{~L}$ & $\mathrm{PM}$ \\
\hline 5 & $\mathrm{~S}$ & $\mathrm{VL}$ & $\mathrm{PS}$ \\
\hline 6 & $\mathrm{~S}$ & $\cdots \cdots$ & $\ldots$ \\
\hline
\end{tabular}

\section{CONFLICT OF INTEREST}

The author confirms that this article content has no conflict of interest.

\section{ACKNOWLEDGEMENTS}

Declared none.

\section{REFERENCES}

H. Rakha, and Y. Zhang, "Sensitivity analysis of transit signal priority impacts on operation of a signalized intersection," Journal of Transportation Engineering, vol. 130, no. 6, pp. 796-804, 2004.

[2] H. Liu, A. Skabardonis, W. B. Zhang, and M. Li, "Optimal detector location for ransit signal priority", Transportation Research Record, 1867, Transportation Research Board, Washington D.C., pp. 144-150, 2004.

[3] Y. Wadjas, and P. G. Furth, "Transit signal priority along an arterial using advanced detection," Transportation Research Record, 1856, Transportation Research Board, Washington D.C., pp.220230, 2003.

[4] P. G. Furth, and B. J. Hemily, T. Muller, and J.G. Strafhman, Uses of archived AVL - APC data to improve transit performance and management: review and potential. Transit Cooperative Research Program Web document 23(Project H-28): Contractor's Final Report, 2003.

[5] H. Liu, Development of Adaptive Transit Signal Priority Systems, Theoretical Approach. Institute of Transportation Studies, Research Report UCB-ITS-PRR-2005, University of California, Berkeley, 2005.

[6] A. P. Davol, Modeling of Traffic Signal Control and Transit Signal Priority Strategies in a Microscopic Simulation Laboratory, Massachusetts Institute of Technology, 2002.

[7] D. Li, Z. Gong, B. An, Y. Ou, W. Cui, and H. Zhu, "A novel security management scheme in wireless sensor networks," International Journal of Digital Content Technology \& its Application, vol. 4, no. 10, pp. 36-44, 2012.
[8] Z. Fan, and X. Wan, "Research of on-demand multipath distance vector routing protocol based on QoS and node movement speed in wireless sensor network," International Journal of Digital Content Technology \& its Application, vol. 4, no. 10, pp. 306-314, 2012.

[9] C. Q. Zhang, and L. Ge, "Using decision tree algorithm to construct intelligent traffic management system, Research of on-demand multipath distance vector routing protocol based on QoS and node movement speed in wireless sensor network," International Journal of Digital Content Technology \& its Application, vol. 4, no. 21, pp. 346-353, 2012.

[10] Y. Lin, "Research on an wireless sensor network streaming media transmission based on the improved Rayleigh wavelet algorithm", Journal of Convergence Information Technology, vol. 7, no. 11, pp. 52-59, 2012.

[11] C. C. Lin, W. P. Chen, S. C. Hsieh, and S. S. Chen, "Design and implementation of ZigBee wireless controller module," Advances in Information Sciences \& Service Sciences, vol. 4, no. 18, pp. 106115, 2012.

[12] O. Xi, D. Li, J. Zhang, H. Liu, H. Zhu, and X. Yang, "Malicious node detection in wireless sensor networks using time series analysis on node reputation," Journal of Convergence Information Technology, vol. 7, no. 15, pp. 8-16, 2012.

[13] J. Li, S. Liu, and S. Wu, "Research of on demand multipath distance vector routing protocol based on QoS and node movement speed in wireless sensor network," International Journal of Digital Content Technology \& its Application, vol. 4, no. 12, pp. 233-240, 2012.

[14] X. J. Qian, D. Fan, C. Chen, Q. Chu, and N. Jiang, "The vehicle positioning system based on the ZigBee technology of wireless sensor networks," Journal of Convergence Information Technology, vol. 8, no. 7, pp. 383-391, 2013.

[15] Y. Lu, and X. He, "Research on key technologies to target coverage algorithm in wireless sensor networks", International Journal of Digital Content Technology \& its Application, vol. 6, no. 17, pp. 542-550, 2012.

[16] X. F. Yang, and Z. Liu, "The application of association rules mining in building intelligent transportation systems", Journal of Convergence Information Technology, vol. 7, no. 20, pp. 575-582, 2012.

(C) Yang Wang; Licensee Bentham Open.

This is an open access article licensed under the terms of the Creative Commons Attribution Non-Commercial License (http://creativecommons.org/licenses/by$\mathrm{nc} / 3.0 /$ ) which permits unrestricted, non-commercial use, distribution and reproduction in any medium, provided the work is properly cited. 